\title{
Les voies sensitives trigéminales : un guide pour l'anesthésie
}

\section{RÉSUMÉ}

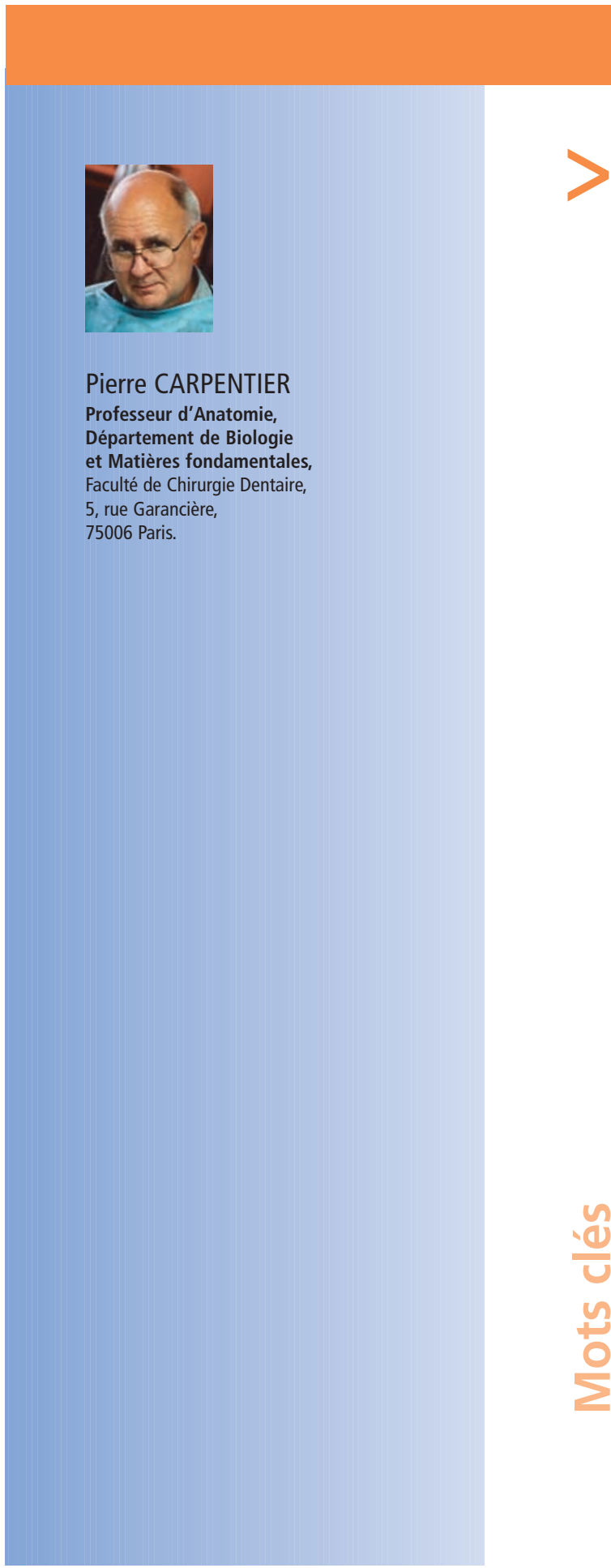

La connaissance des trajets nerveux est un facteur déterminant pour réussir dans les meilleures conditions une anesthésie locale dans la cavité orale. Les données de base concernant l'innervation trigéminale sont exposées en utilisant la nouvelle terminologie anatomique internationale. La documentation est réalisée à partir de photographies d'os sec sur lesquelles sont reproduits les principaux trajets nerveux. Des images de dissection et des coupes anatomiques viennent compléter les schémas. Cet article illustre le concept d'anatomie clinique, car il est volontairement limité aux seuls éléments nécessaires au clinicien pour comprendre et pratiquer l'anesthésie locale.

Cet article, déjà paru dans le numéro spécial "Anesthésie» des AOS ( $\left.n^{\circ} 179\right)$ en 1992 méritait, par sa qualité scientifique et iconographique, une nouvelle publication susceptible d'intéresser nos confrères dans leur exercice quotidien

Le comité de rédation

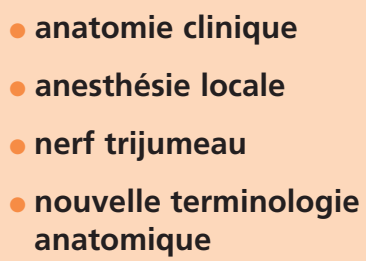




\section{Introduction}

$>$

L'anesthésie locale est aujourd'hui considérée par le praticien et le patient comme une étape indispensable à la réalisation d'un grand nombre d'actes ayant pour site la cavité orale. Les solutions anesthésiques actuelles sont d'une grande efficacité et procurent, à faible dose, une analgésie de qualité, à condition toutefois qu'elles soient déposées à proximité du tronc nerveux, en particulier lorsque celui-ci est de gros calibre.

Dès lors, la connaissance des trajets nerveux principaux, ainsi que des suppléances, devient un facteur déterminant pour réussir dans les meilleures conditions une anesthésie locale.
La description des voies sensitives trigéminales a fait l'objet de nombreuses publications dans des ouvrages et revues anatomiques qui ne font pas obligatoirement partie des lectures habituelles du praticien[7-9, 13, 16-18]. II est donc intéressant de rassembler ici un certain nombre de données et d'en profiter pour introduire la Nouvelle Terminologie Anatomique internationale (N.T.A.). Pour ne pas dérouter le lecteur et favoriser l'apprentissage de ce nouveau langage, nous garderons entre parenthèses la dénomination propre à l'école française. Il est important que les cliniciens se familiarisent progressivement à cette nouvelle terminologie, car elle est utilisée dans tous les ouvrages récents d'anatomie et d'imagerie.

\section{Les branches du nerf trijumeau}

C'est à partir du ganglion trigéminal (Gasser) que vont se différencier les trois branches de division du nerf trijumeau, nerf branchial du premier arc (fig. 1) : les nerfs ophtalmique V1, maxillaire V2 (maxillaire supérieur) et mandibulaire V3 (maxillaire inférieur), le seul à contenir des fibres efférentes destinées principalement aux muscles masticateurs.

\section{Le nerf ophtalmique V1}

Le territoire du V1 concerne peu l'odontologiste ; nous rappellerons simplement que ce nerf se divise à l'intérieur du sinus caverneux en trois branches terminales - lacrymale, frontale et nasociliaire (nasale) -, qui traversent la fissure orbitaire supérieure (fente sphénoïdale). Le nerf frontal se divise ensuite en deux rameaux (supra-orbitaire et supra-trochléaire) qui se distribuent aux téguments du front, de la racine du nez et de la paupière supérieure. Le nerf lacrymal est destiné à la glande lacrymale et à la partie latérale de la paupière supérieure. Le nerf naso-ciliaire donne des branches au bulbe oculaire, au ganglion ciliaire, et participe à l'innervation des muqueuses de la paroi latérale des cavités nasales et des sinus ethmoïdaux, frontaux et sphénoïdaux, par l'intermédiaire des nerfs ethmoïdaux antérieur et postérieur (fig. 2). Le V1 est un nerf essentiellement sensitif. 


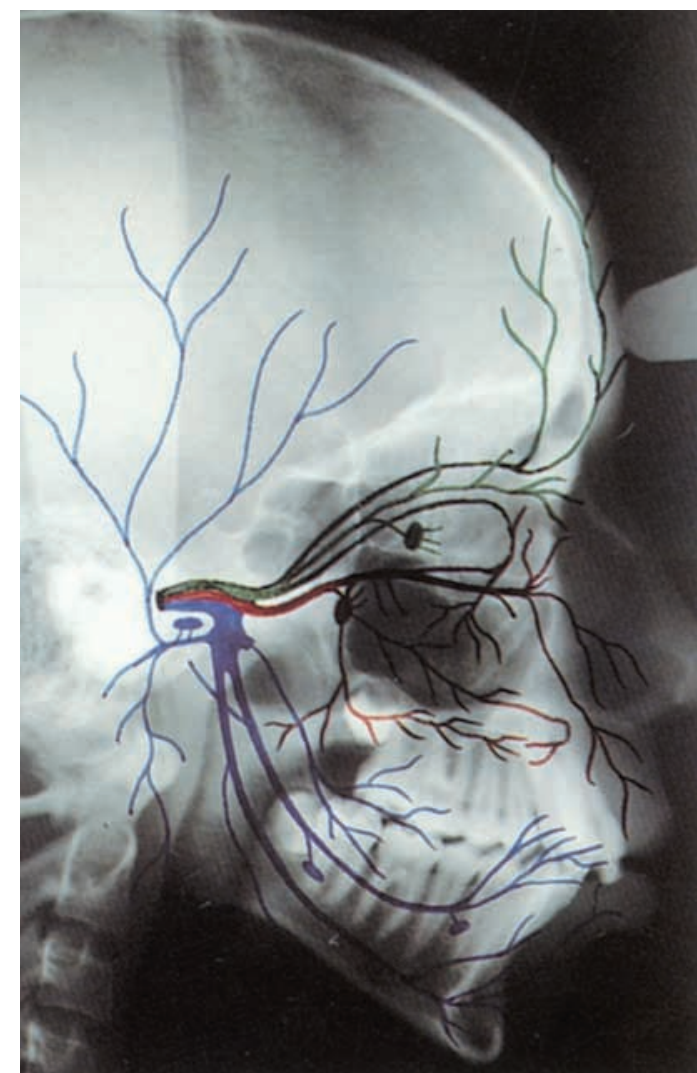

fig. 1 Projection des principales branches trigéminales sur une téléradiographie de profil. En vert le nerf ophtalmique (V1) en rouge le nerf maxillaire (V2), en bleu le nerf mandibulaire (V3).

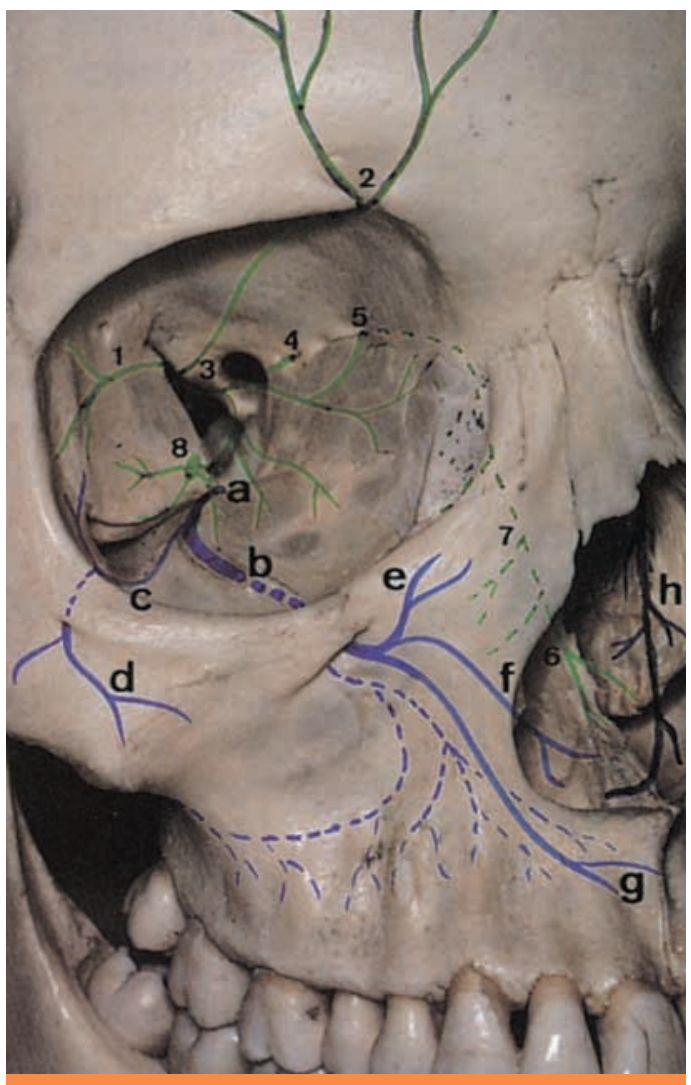

fig. 2 Branches de division du nerf ophtalmique en vert, et du nerf maxillaire en bleu. 1 : nerf lacrymal ; 2 : nerf frontal ; $3:$ nerf naso-ciliaire ; $4:$ nerf ethmoïdal postérieur ; 5 : nerf ethmoïdal antérieur ; 6 : nerf naso-lobaire $; 7:$ rameaux nasaux internes ; 8 : ganglion ciliaire ; a : nerf maxillaire ; b : nerf infra-orbitaire $c$ : rameau orbitaire ; d : rameau zygomatico-facial ; e : rameaux palpébraux ; $f:$ rameaux nasaux ; $g$ : rameaux labiaux ; $\mathrm{h}:$ nerf naso-palatin.

\section{Le nerf maxillaire $\mathrm{V} 2$}

Il traverse la base du crâne en empruntant le trou rond (grand rond) et débouche dans la fosse ptérygo-palatine (arrière-fond de la fosse ptérygo-maxillaire), dont il se dégage en réalisant un premier coude en direction latérale, puis un second vers l'avant à travers la fissure orbitaire inférieure (fente sphéno-maxillaire). À ce niveau, le nerf s'engage dans le sillon et le canal infra-orbitaire situés dans le plancher de l'orbite. Il devient alors le nerf infra-orbitaire. Lorsqu'il émerge sur la face ventro-latérale du maxillaire à travers le foramen infra- 


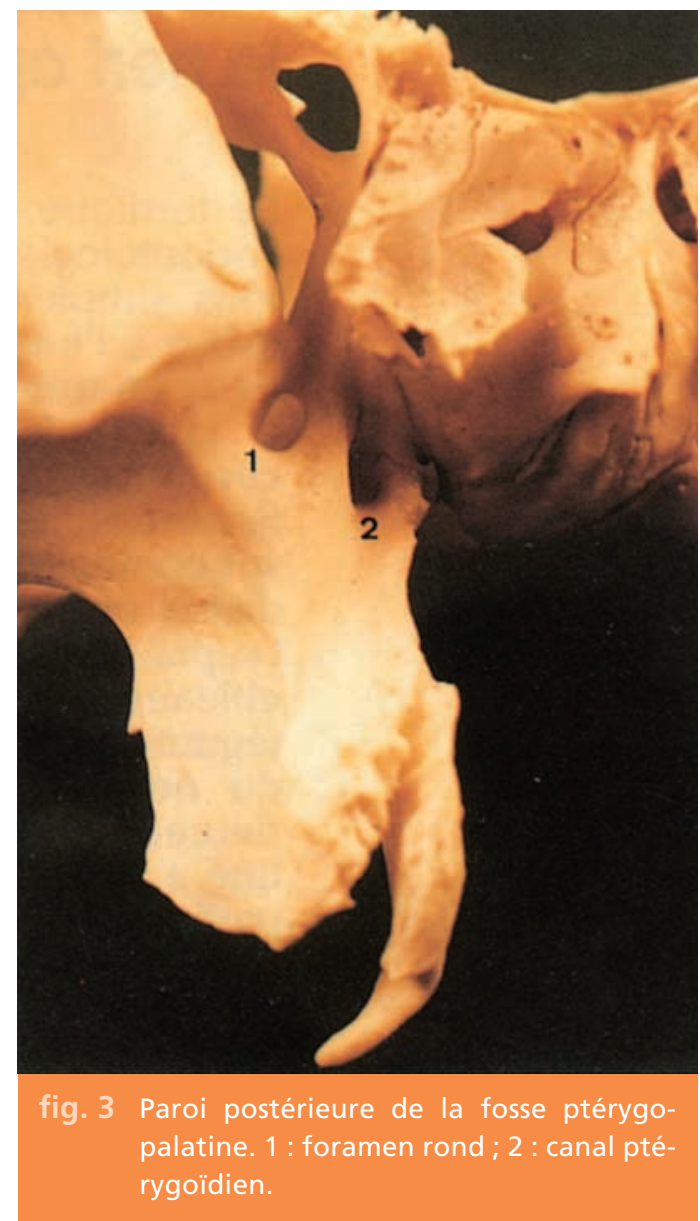

orbitaire, le nerf se divise en rameaux palpébraux, nasaux et labiaux (fig. 2).

Si on peut décrire le trajet du nerf maxillaire en se référant essentiellement aux structures osseuses, les communications de la fosse ptérygo-palatine avec les cavités bordantes permettent aussi d'appréhender la destination de ses diverses branches collatérales. Immédiatement en dedans du foramen rond, débouche le canal ptérygoïdien (canal vidien), délimité par l'implantation des deux lames du processus ptérygoïde sur le corps du sphénoïde et la partie attenante de la grande aile (fig. 3). À travers celui-ci chemine le nerf ptérygoïdien (vidien), qui comprend des fibres efférentes viscérales générales parasympathiques et sympathiques.
Les premières issues du noyau lacrymo-palatonasal (muco-lacrymo-nasal) sont associées, à leur origine, aux nerfs facial et glosso-pharyngien dont elles empruntent des branches collatérales : les nerfs pétreux. Ceux-ci émergent dans l'endocrâne, fusionnent et se dirigent vers le foramen déchiré. C'est à ce niveau que les fibres sympathiques issues du plexus périartériel et la carotide interne les rejoignent pour constituer le nerf ptérygoïdien qui traverse le foramen déchiré (trou déchiré antérieur), emprunte le canal ptérygoïdien et se termine dans le ganglion ptérygo-palatin (sphéno-palatin) situé dans la fosse homonyme. Seules les fibres parasympathiques font synapses dans ce ganglion puisque les fibres sympathiques ont déjà fait synapses dans le ganglion cervical supérieur.

C'est ainsi que, par l'intermédiaire des anastomoses entre le ganglion ptérygo-palatin et le nerf maxillaire, ce dernier s'enrichit de fibres postganglionnaires sympathiques et parasympathiques destinées à l'innervation des nombreuses glandes disséminées dans les muqueuses nasale et palatine, ainsi qu'à la glande lacrymale.

Le foramen sphéno-palatin qui met en communication la fosse ptérygo-palatine avec la cavité nasale, occupe une position cranio-dorsale par rapport à l'extrémité du cornet moyen. C'est par cette voie que le nerf sphénopalatin traverse la paroi latérale des cavités nasales et différencie les nerfs nasaux supérieurs et naso-palatin (fig. 4).

Les premiers sont destinés à l'innervation de la paroi latérale, des cornets et des méats. Le nerf ethmoïdal antérieur, branche du nerf nasociliaire $(\mathrm{VI})$, contrôle la partie antérieure de cette paroi.

Le nerf naso-palatin gagne la cloison nasale, donne des rameaux à la muqueuse qui la tapisse, puis se termine par le rameau palatin 


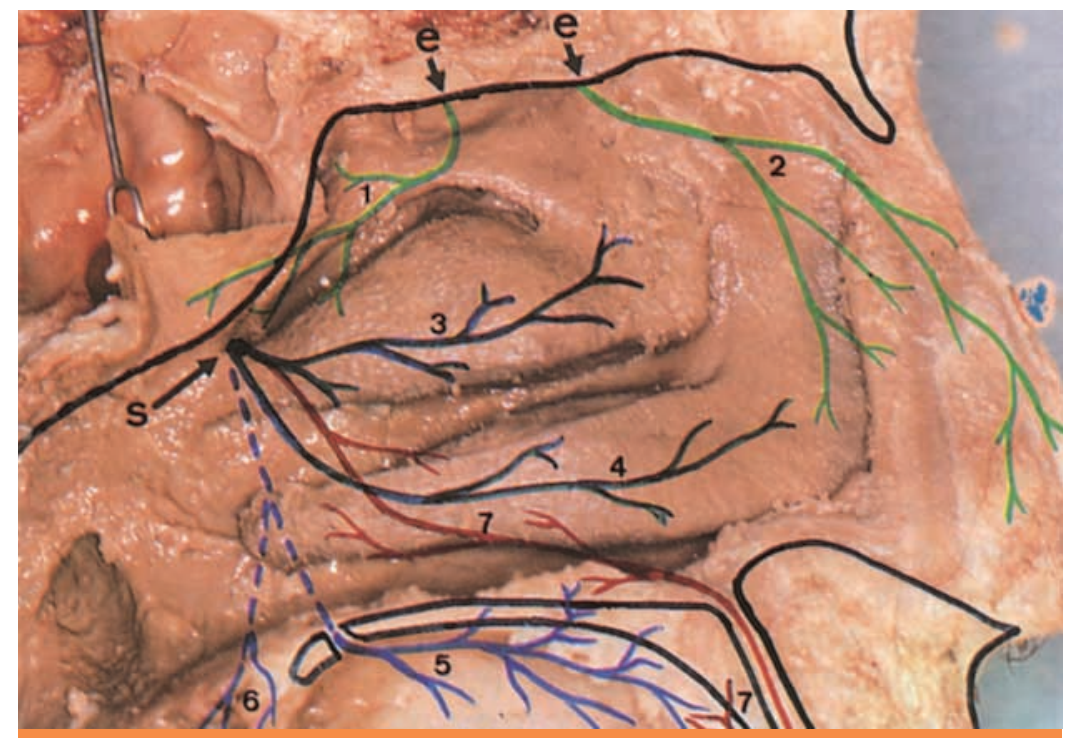

fig. 4 Innervation de la paroi latérale de la cavité nasale. e : foramens ethmoïdaux ; $\mathrm{s}$ : foramen sphéno-palatin. En vert : 1 : nerf ethmoïdal postérieur ; 2 : nerf ethmoïdal antérieur, branches du V1. En bleu : $3:$ nerfs nasaux supérieurs ; $4:$ nerf nasal inférieur ; $5:$ nerf grand palatin ; 6 : nerfs petit palatin et palatin accessoire. En rouge : 7 : projection sur la paroi latérale du nerf naso-palatin qui descend le long de la cloison nasale.

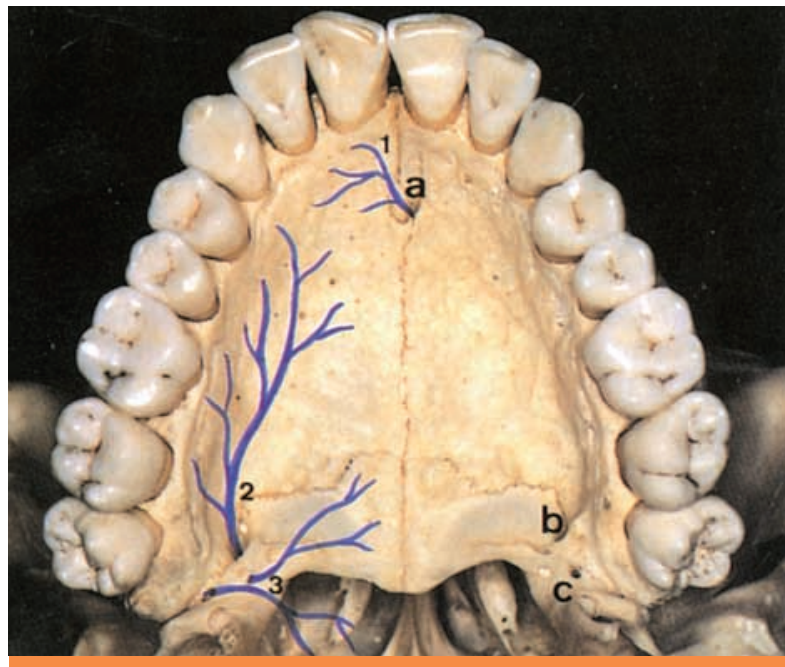

fig. 5 Innervation du palais dur. 1 : rameau palatin du nerf naso-palatin ; 2 : nerf grand palatin ; $3:$ nerf petit palatin et palatin accessoire ; $a$ : foramen incisif ; $b$ : foramen grand palatin ; $c$ : foramens petits palatins.

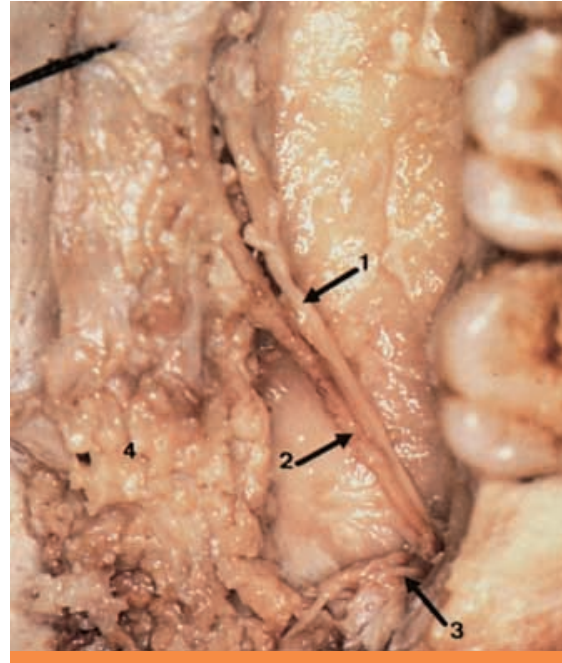

fig. 6 Décollement de la muqueuse du palais dur. 1 : nerf grand palatin ; 2 : artère grande palatine (branche profonde principale) ; 3 : nerfs petits palatins : 4 : glandes salivaires palatines. 
qui emprunte le canal incisif et débouche dans la cavité orale pour innerver le secteur incisivocanin de la muqueuse palatine (fig. 5).

La fosse ptérygo-palatine est également en communication avec la cavité orale par l'intermédiaire du canal grand palatin (palatin postérieur) situé dans l'épaisseur de la paroi latérale des cavités nasales. Cette voie est empruntée par les nerfs palatins. Le nerf grand palatin (palatin antérieur) parcourt ce canal en compagnie de l'artère palatine descendante qui, au niveau du foramen grand palatin, devient l'artère grande palatine (fig. 6). Celleci se divise en deux ou trois branches, dont une principale qui chemine en compagnie du nerf à la face profonde de la muqueuse du palais dur. Le nerf se distribue à toute cette muqueuse à l'exception du secteur incisivocanin contrôlé par le rameau palatin du nerf naso-palatin. L'artère, par contre, vascularise toute la muqueuse du palais dur, puis emprunte le canal incisif en direction des cavités nasales où elle se termine.

Les nerfs petits palatins et accessoires, qui se partagent avec des branches du IX I'innervation sensitive du voile, se différencient dans le canal grand palatin, empruntent des canalicules qui traversent le processus pyramidal du palatin et débouchent par les foramens petits palatins. (La N.T.A. emprunte le singulier de Foramina et introduit le marqueur français du pluriel «s»).

Rappelons que tous les nerfs palatins contiennent des fibres efférentes viscérales générales destinées aux glandes salivaires palatines.

Par la voie du canal grand palatin, il est possible de réaliser un bloc anesthésique du V2 en déposant la solution dans la fosse ptérygopalatine.

Cette technique, comme toutes celles qui nécessitent la pénétration d'un foramen, a l'inconvénient d'entraîner des lésions vasculaires et/ou nerveuses, et ne peut être de ce fait utilisée de façon routinière. Sa mise en œuvre demande une certaine habitude pour repérer le foramen grand palatin et l'axe du canal. L'aiguille ne doit être forcée en aucun cas, sous peine de perforer la fragile paroi canalaire. La profondeur moyenne de pénétration est de l'ordre de $30 \mathrm{~mm}$ et l'aspiration est impérative compte tenu des vaisseaux maxillaires (internes).

La fosse ptérygo-palatine communique avec la fosse infra-temporale (ptérygo-maxillaire) par la fissure (fente) ptérygo-maxillaire. Cette voie est empruntée par le V2 qui, avant de s'engager dans la fissure orbitaire inférieure, évolue au contact du bord supérieur de la tubérosité maxillaire et détache les nerfs alvéolaires supérieurs postérieurs (dentaires) (fig. 7). Ils se dirigent en bas et en avant, longeant dans de fines gouttières la tubérosité, pénètrent les orifices alvéolaires postéro-supérieurs et forment un plexus qui innerve :

- les trois molaires (pulpe et desmodonte) ;

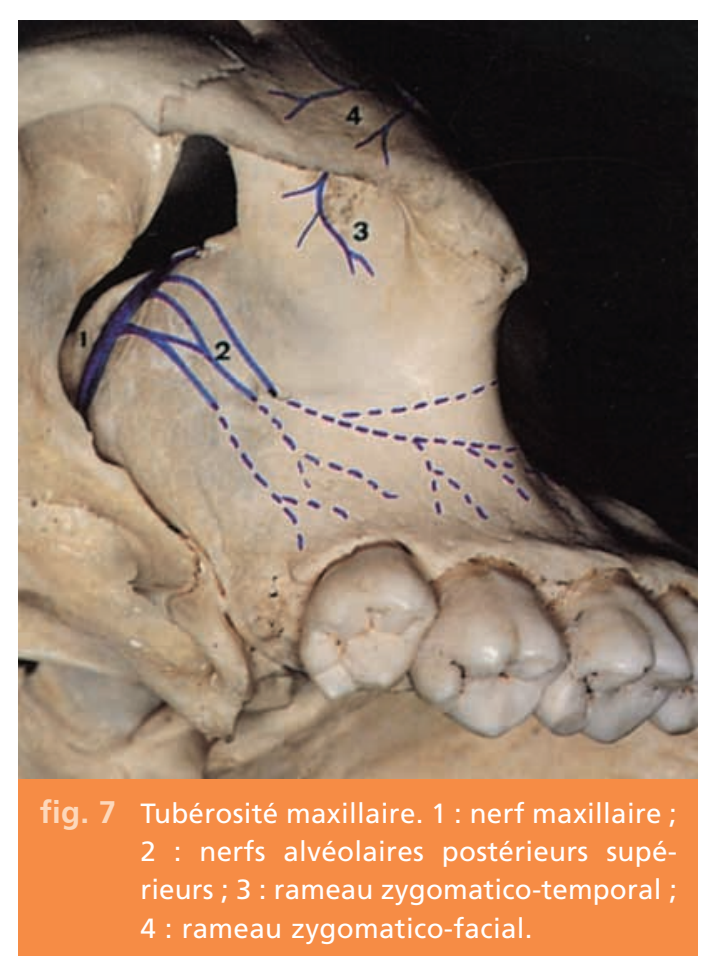


- la paroi externe de l'os maxillaire du secteur molaire ;

- la muqueuse de la partie dorso-latérale du sinus maxillaire.

L'une des branches alvéolaires postérieures ne pénètre pas l'os mais gagne la gencive et la muqueuse vestibulaire de la région molaire (fig. 8). Dans un article consacré à l'anesthésie tubérositaire, cette région est l'objet d'une description détaillée[1].

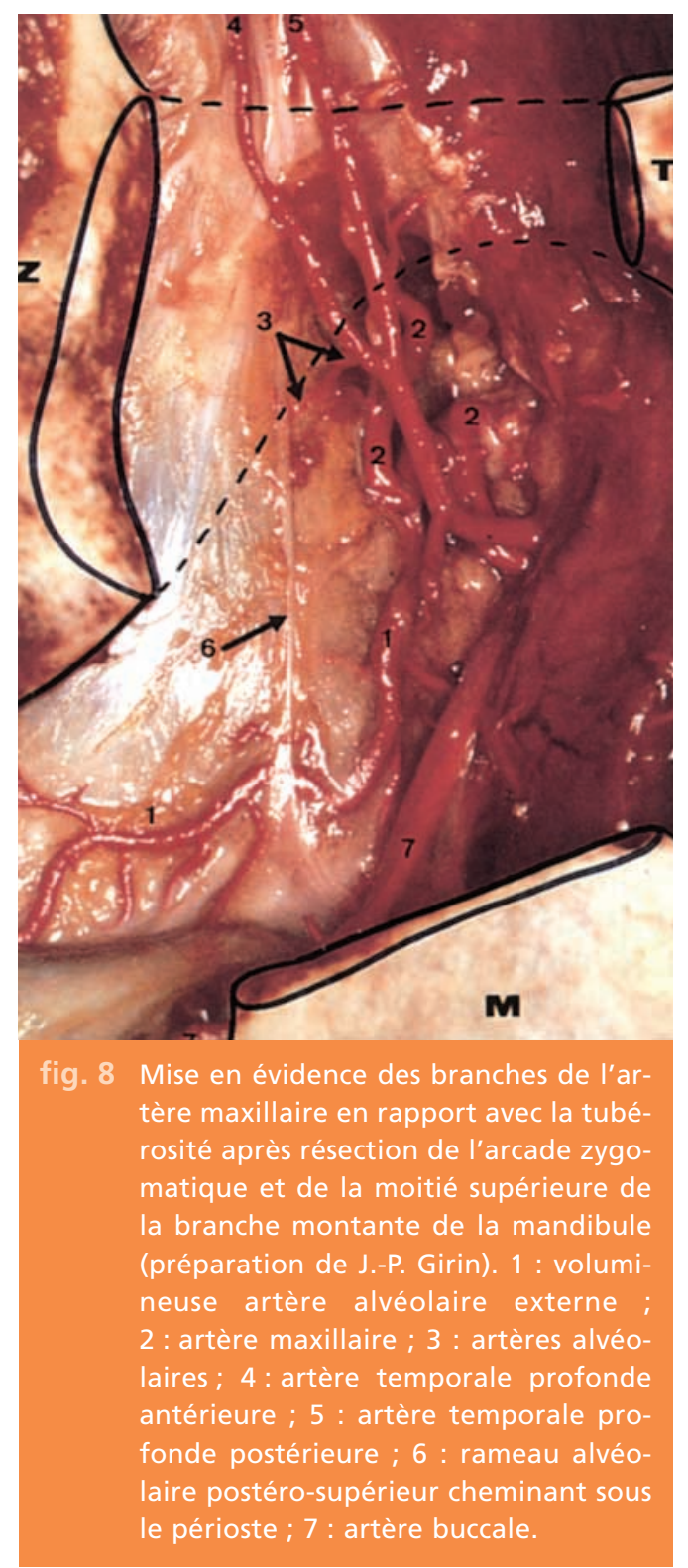

Le nerf alvéolaire supérieur moyen, destiné aux prémolaires, se détache du nerf infra-orbitaire au cours de son trajet intracanalaire. Dans $50 \%$ des cas, ce nerf fait défaut et les prémolaires sont alors innervées par les nerfs alvéolaires antérieurs supérieurs (fig. 9). Ceuxci se détachent environ $5 \mathrm{~mm}$ avant le débouché du canal, au foramen infra-orbitaire, ce qui permet leur blocage par une injection à proximité ou à l'intérieur de cet orifice (fig. 9). Cette technique, non dépourvue de risques, nous paraît sans réel intérêt puisqu'il est possible d'anesthésier valablement les prémolaires et le groupe incisivo-canin en pratiquant des injections para-apicales hautes. La paroi ventro-latérale du maxillaire, compte tenu de sa minceur, laisse percevoir en surface les canalicules osseux abritant les rameaux nerveux (fig. 10). À l'aplomb des prémolaires, on s'assurera par un test d'aspiration que l'aiguille n'a pas pénétré la veine faciale qui est en rapport constant avec la fosse canine. On évitera également, lors d'une anesthésie de l'incisive médiale (centrale), le tissu fibreux qui attache le cartilage de la cloison sur l'épine nasale antérieure, car cette zone est toujours douloureuse à l'injection (fig. 11). II ne faut cependant pas trop déporter latéralement le point d'impact de l'aiguille, car le rameau de l'incisive médiale est proche du plan sagittal médian et peut être anastomosé avec le nerf naso-palatin. En présence d'un échec anesthésique de cette dent ou de ce secteur, on doit envisager un complément de ce rameau, soit dans le canal incisif, soit au niveau du plancher nasal.

Le nerf infra-orbitaire se termine en se divisant en plusieurs branches destinées à la paupière inférieure, à l'aile du nez, à la partie antérieure de la joue et à la lèvre supérieure. L'anesthésie de ces territoires est fréquente lors d'une injection para-apicale du bloc incisivo-canin. 


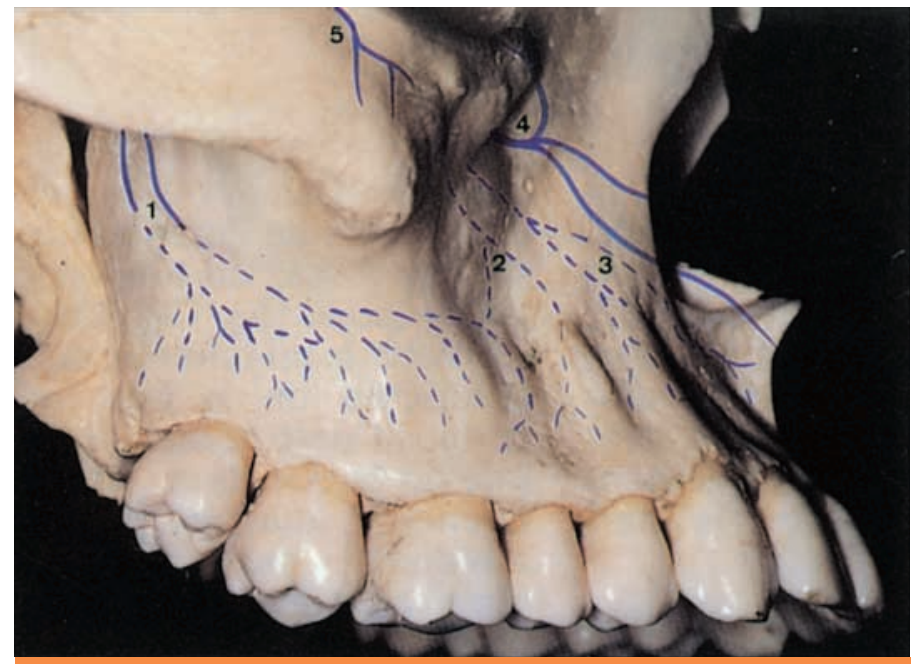

fig. 91 : nerfs alvéolaires postéro-supérieurs ; 2 : nerf alvéolaire supérieur moyen ; 3 : nerfs alvéolaires antéro-supérieurs ; 4 : nerf infra-orbitaire ; 5 : rameau zygomaticofacial.

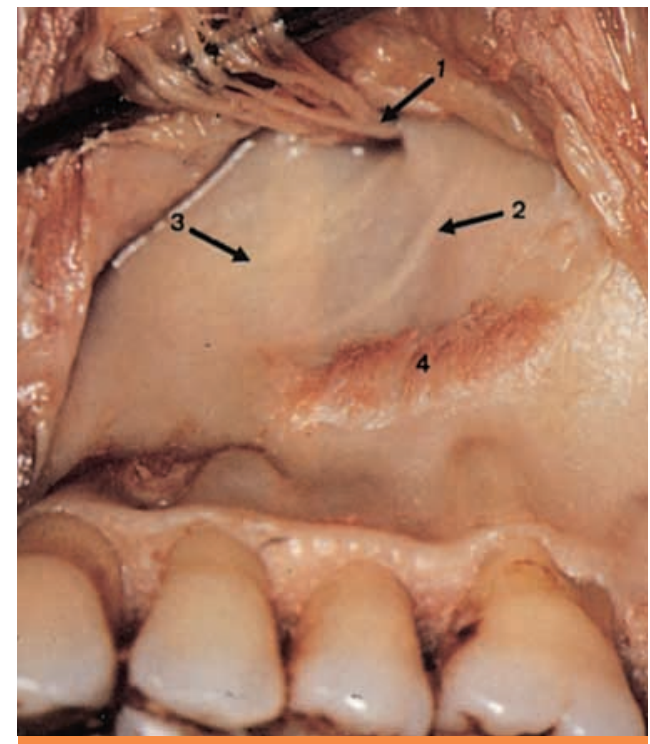

fig. 10 Paroi ventro-latérale du maxillaire. 1 : nerf infra-orbitaire à sa sortie du canal ; 2 : nerf alvéolaire supérieur moyen ; 3 : nerf alvéolaire supérieur antérieur ; 4 : insertion du muscle releveur de l'angle de la bouche (canin).

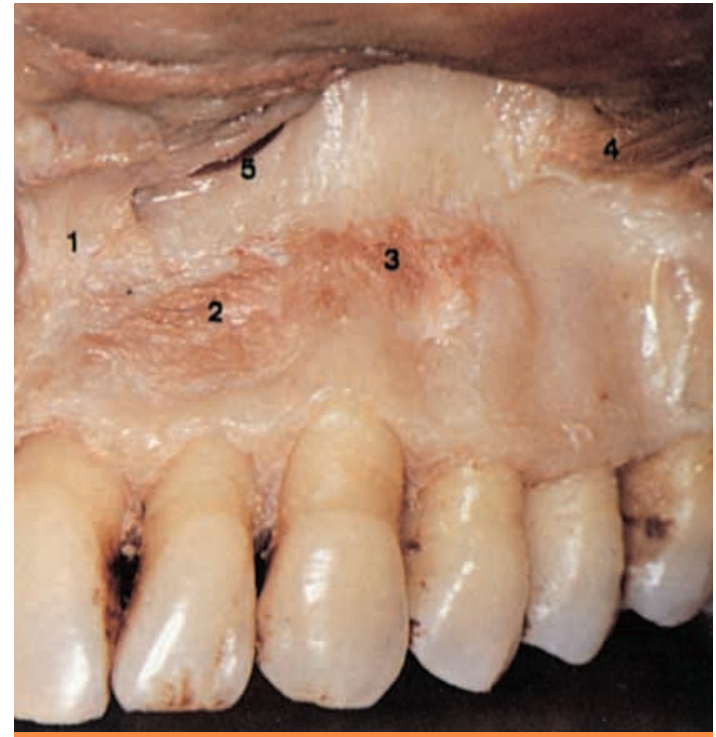

fig. 11 Éléments en rapport avec la pointe de l'aiguille dans le secteur antérieur maxillaire. 1 : tissu fibreux amarrant le cartilage de la cloison à l'épine nasale antérieure ; 2 : aire d'insertion du muscle abaisseur du septum nasal (myrtiforme) ; 3 : aire d'insertion du muscle incisif supérieur ; 4 : muscle abaisseur de l'angle de la bouche ; 5 : orifice narinaire. 


\section{Le nerf mandibulaire V3}

Le nerf mandibulaire sort de la base du crâne par le foramen ovale et se divise classiquement en deux troncs (fig. 12). Le tronc terminal antérieur qui donne des branches pour les muscles temporal, masséter et ptérygoïdien latéral (externe), possède également une branche sensitive : le nerf buccal. Celui-ci descend en compagnie de l'artère homonyme sous le fascia du tendon profond du temporal (fig. 13). Arrivé sensiblement à mi-hauteur de la face antérieure de la branche montante, le nerf rejoint la face latérale du buccinateur, dont la partie inférieure attachée à la branche montante par des éléments fibreux est au contact du tendon profond[13]. Le nerf, en se dirigeant vers l'avant, décrit une courbe à concavité supérieure et se divise en de nombreuses

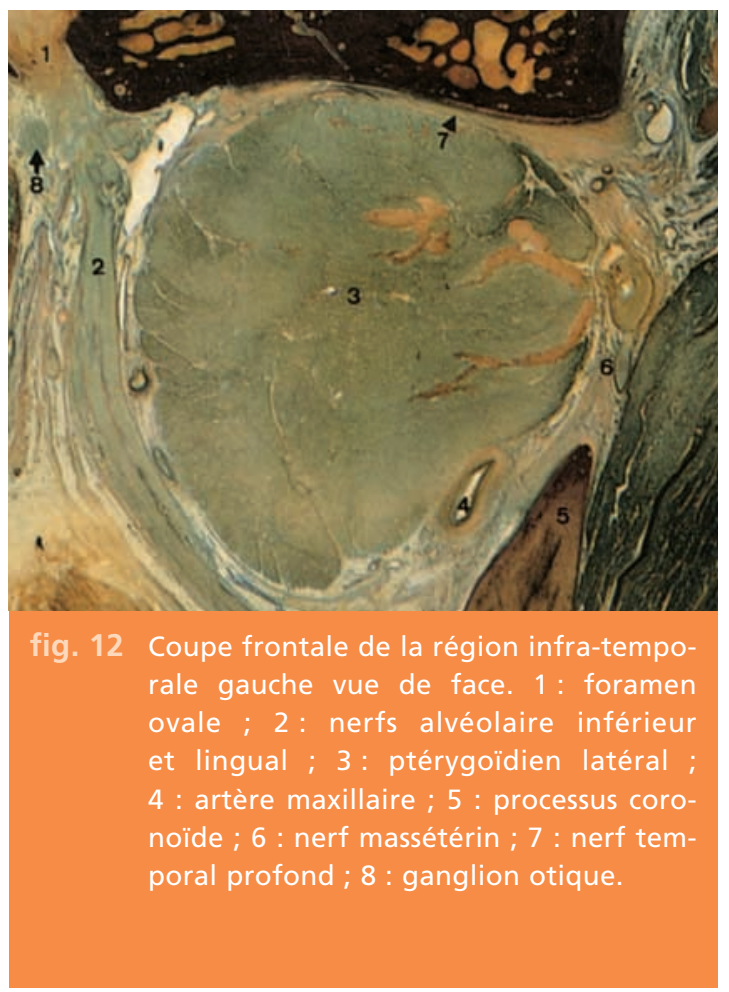

branches qui traversent le muscle pour gagner la muqueuse jugale, la gencive et la muqueuse alvéolaire du secteur molaire vestibulaire. Comme nous l'avions déjà signalé[3, 4, 11, 12], considérant des études fondamentales[5] et cliniques[15], ce nerf peut participer à l'innervation des molaires mandibulaires, et son blocage paraît opportun au-delà de la stricte indication chirurgicale de ce secteur. On choisira de préférence l'anesthésie du tronc nerveux en prenant comme repère vertical la partie la plus profonde du bord antérieur de la branche montante et en piquant dans la fosse rétromolaire en dehors de la crête temporale.

Le tronc terminal postérieur se divise en quatre branches : le nerf alvéolaire inférieur (dentaire), le nerf lingual, l'auriculo-temporal et le

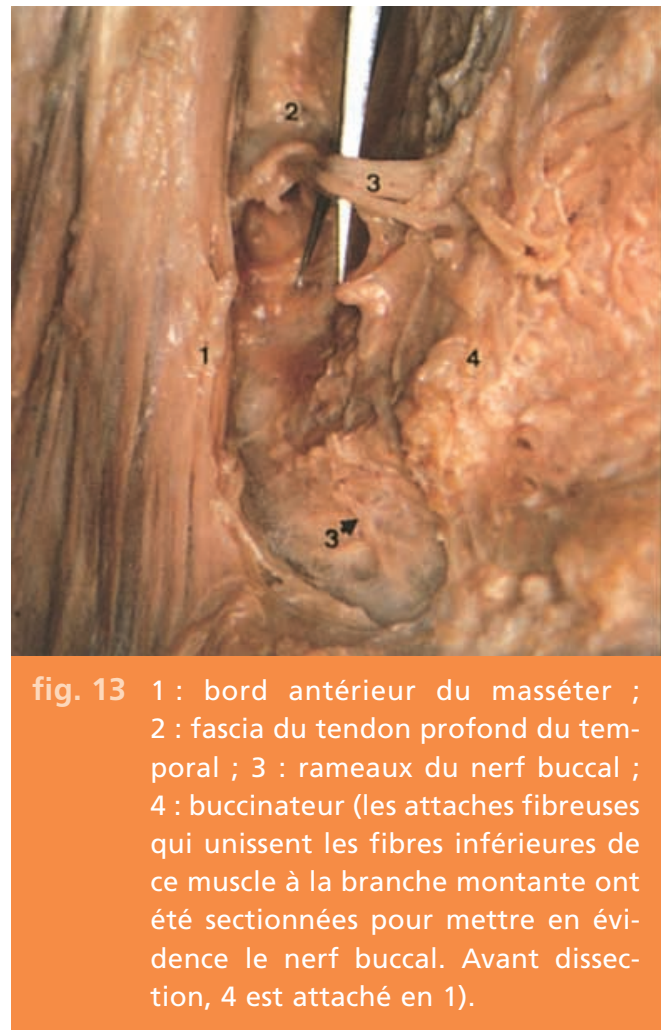

Actualités Odonto-Stomatologiques - $\mathrm{n}^{\circ} 243$ - septembre 2008 


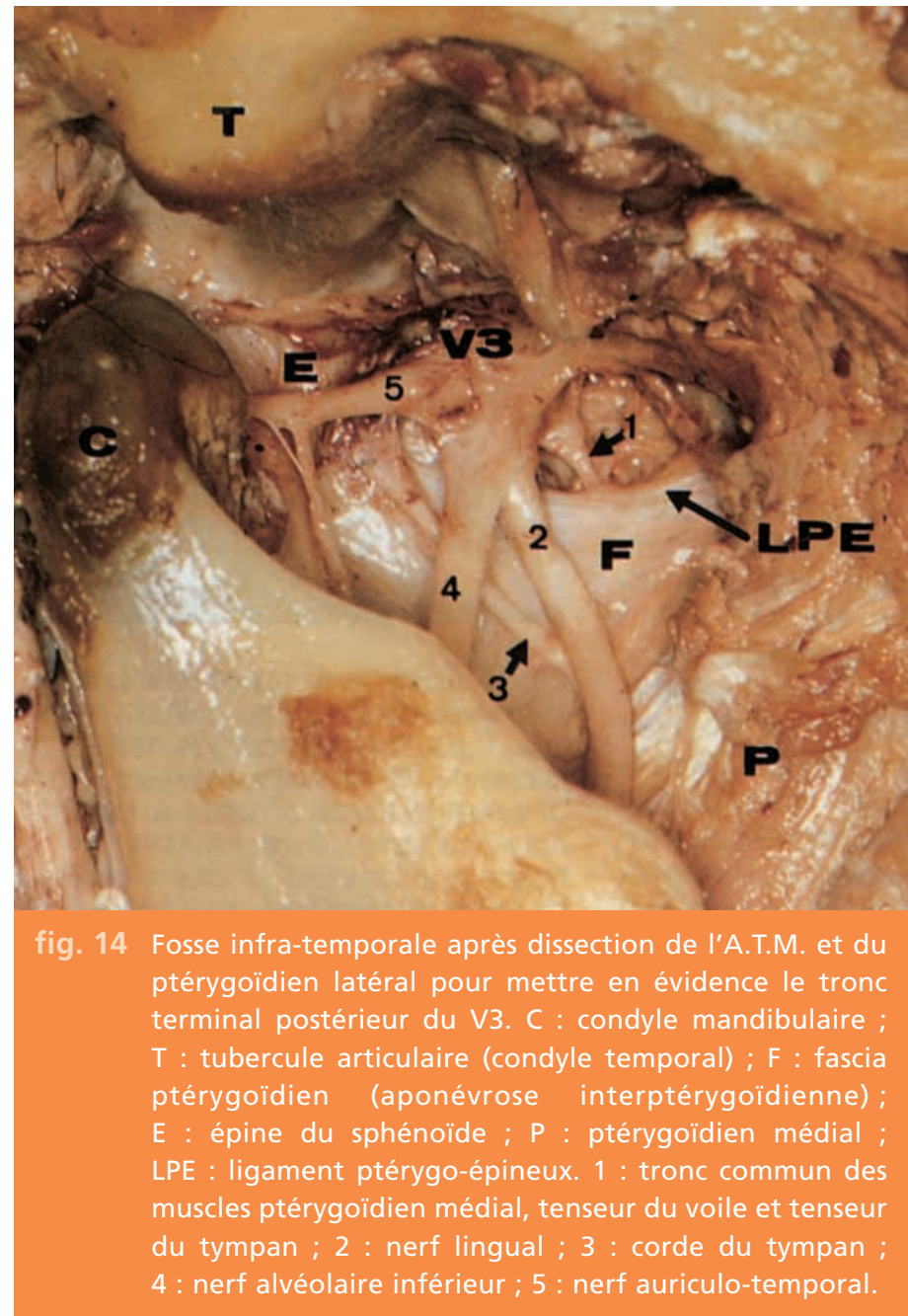

tronc commun des muscles ptérygoïdien médial (interne), tenseur du voile (péristaphylin externe) et tenseur de la membrane du tympan (muscle du marteau) (fig. 14).

\section{Le nerf alvéolaire inférieur}

Le nerf alvéolaire inférieur est celui dont le blocage intéresse tout particulièrement I'odonto-stomatologiste. II contrôle :

- toutes les dents mandibulaires (pulpe et desmodonte) ;

- l'os mandibulaire (corps et partie inférieure de la branche montante) ;
- la gencive et la muqueuse alvéolaire vestibulaire en avant du foramen mentonnier ainsi que la muqueuse labiale inférieure ;

- les téguments de la lèvre inférieure et du menton.

Le trajet et les rapports du nerf alvéolaire inférieur dans la région infratemporale sont déterminants pour la compréhension de l'anesthésie tronculaire à l'épine de Spix. À son origine, le nerf est situé en dedans du muscle ptérygoïdien latéral et en dehors du fascia ptérygoïdien (aponévrose interptérygoïdienne). II se dirige en bas, en dehors, et légèrement en avant, pour gagner l'échancrure mandibulaire bordée 
par la lingula et I'antilingula. Lorsque le nerf approche cette zone, il est contenu dans du tissu graisseux qui comble l'espace ménagé entre :

- le tendon profond du temporal et la face interne de la branche montante, latéralement ;

- le ptérygoïdien médial et le ligament sphéno-mandibulaire, médialement (fig. 15).

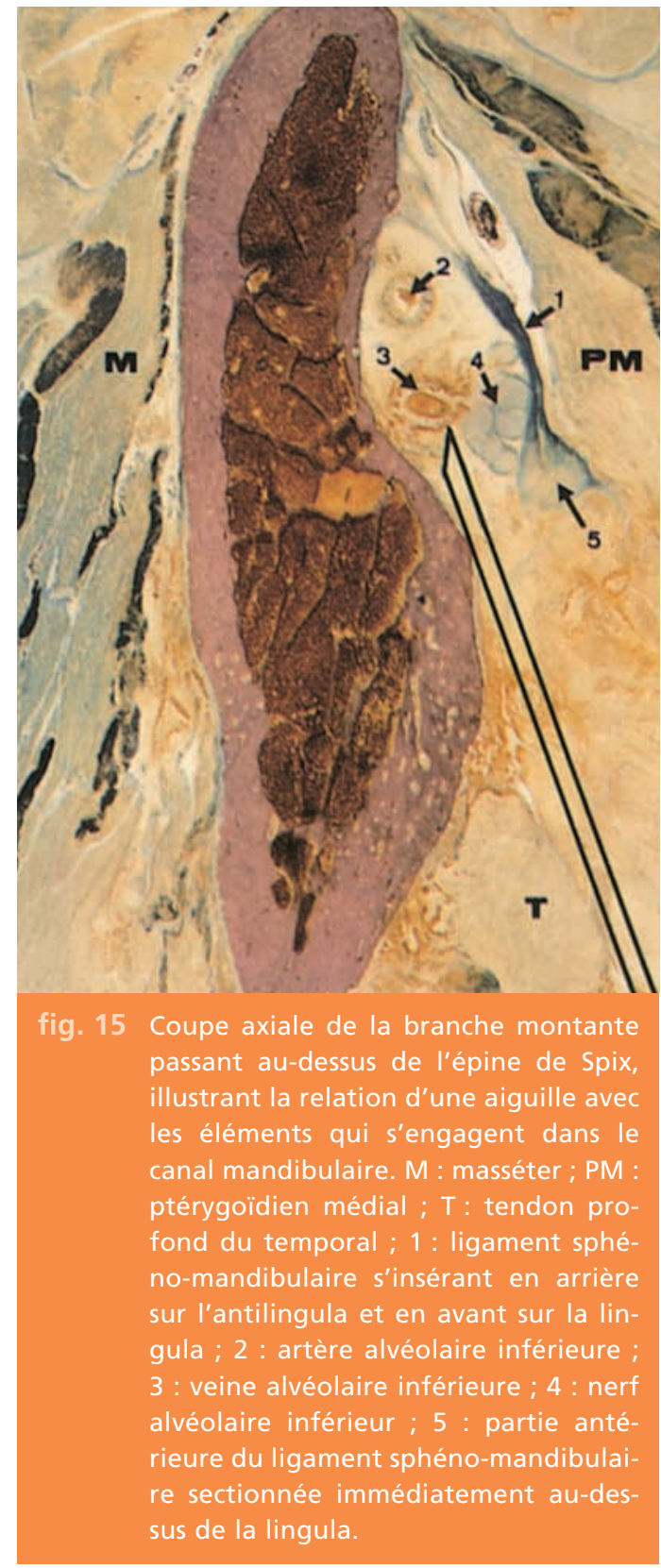

Il est en rapport avec l'artère alvéolaire inférieure en arrière, et le nerf lingual situé environ $1 \mathrm{~cm}$ en avant.

Le nerf alvéolaire inférieur détache, avant de s'engager dans le canal, le nerf du mylo-hyoïdien et du ventre antérieur du digastrique qui s'engage dans une gouttière osseuse, transformée en canal par l'extension de l'insertion du ligament sphéno-mandibulaire (fig. 16). Longtemps considéré comme un nerf typiquement moteur, il contient en réalité $20 \%$ de fibres afférentes et participerait à l'innervation de l'os, voire des dents mandibulaires par l'intermédiaire de rameaux qui pénètrent le corps mandibulaire[6, 10, 14, 19].

Dans le canal mandibulaire, de l'échancrure à la racine mésiale de la deuxième molaire, le nerf alvéolaire inférieur est au-dessus de l'artère, puis celle-ci le contourne pour se placer progressivement au-dessus de lui. Les travaux de Carter et Keen ont mis en évidence les différentes conformations du nerf alvéolaire inférieur[5]. Dans le type I, le plus fréquent, il se présente sous la forme d'un tronc unique situé dans un canal en étroit rapport avec les apex des molaires. Les rameaux dentaires et desmodontaux sont alors très courts et directs. Dans le type II, le canal étant situé à distance des apex, ces rameaux se détachent plus dorsalement, ils sont plus longs et présentent des anastomoses. Dans le type III, qui représente environ un tiers des cas, le nerf différencie deux branches dès sa pénétration dans le canal. L'une supérieure, destinée à l'os, à la pulpe et au desmodonte des molaires et prémolaires, peut être considérée comme une branche alvéolaire. L'autre, plus volumineuse, contient les nerfs mentonnier et incisif. Quel que soit le type rencontré, on retrouve des rameaux perforants qui émergent à travers l'os cortical de la fosse rétro-molaire et du vestibule en regard des molaires et que I'on bloque en réalisant une anesthésie du nerf buccal (fig. 17). 


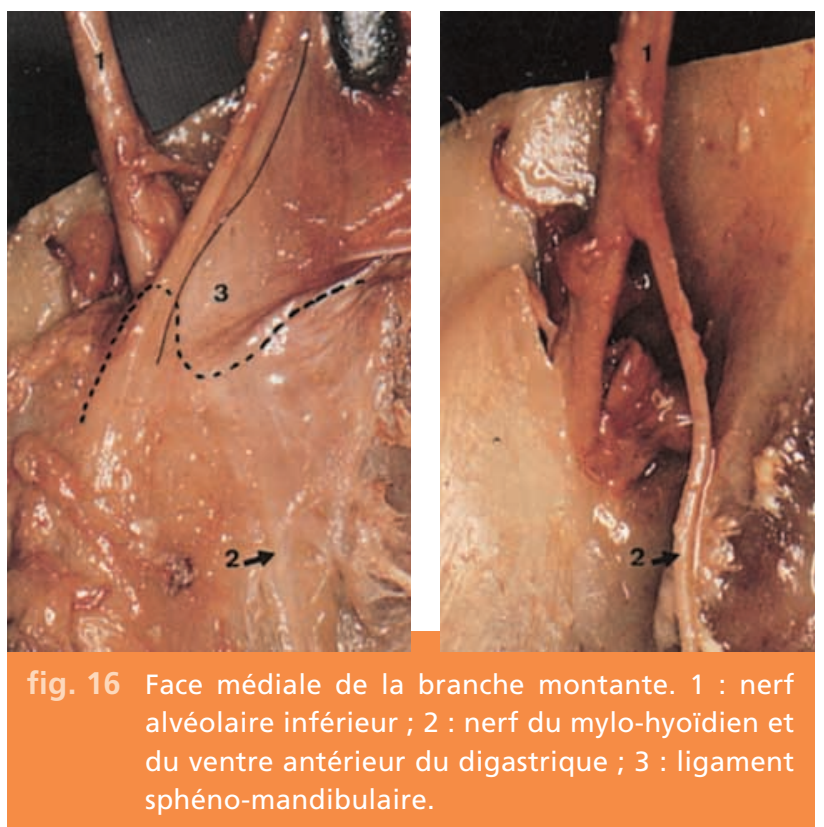

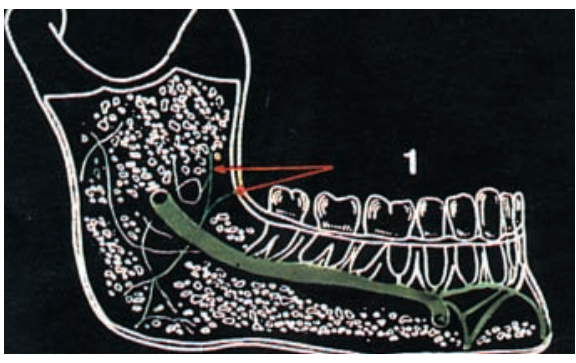
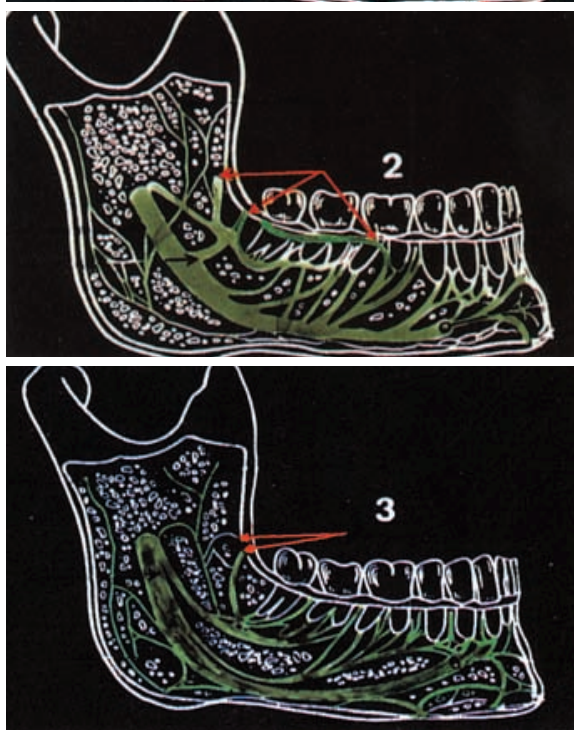

fig. 17 Distribution intramandibulaire du nerf alvéolaire inférieur selon Carter et Keen. Les rameaux perforants sont indiqués par des flèches rouges.
Classiquement, le nerf alvéolaire inférieur se termine en différenciant deux branches :

- le nerf incisif qui poursuit son trajet à travers I'os trabéculaire jusqu'à la symphyse, tout en donnant des rameaux pour la première prémolaire et le groupe incisivo-canin ;

- le nerf mentonnier qui se divise à la sortie du foramen en plusieurs branches destinées au vestibule, à la lèvre et au menton (fig. 18 et 19). La topographie du nerf mentonnier ayant fait l'objet d'un récent article, nous conseillons au lecteur soucieux d'en savoir plus de s'y reporter[2].

La pénétration du foramen mentonnier avec une aiguille est source de lésion vasculaire et donc d'hématome toujours fort disgracieux dans cette région. L'anesthésie para-apicale du groupe incisivo-canin est efficace, sous réserve de ne pas trop enfoncer l'aiguille dans le vestibule, afin d'éviter la protubérance mentonnière dont l'épaisseur d'os cortical est un obstacle à la diffusion de la solution anesthésique. 

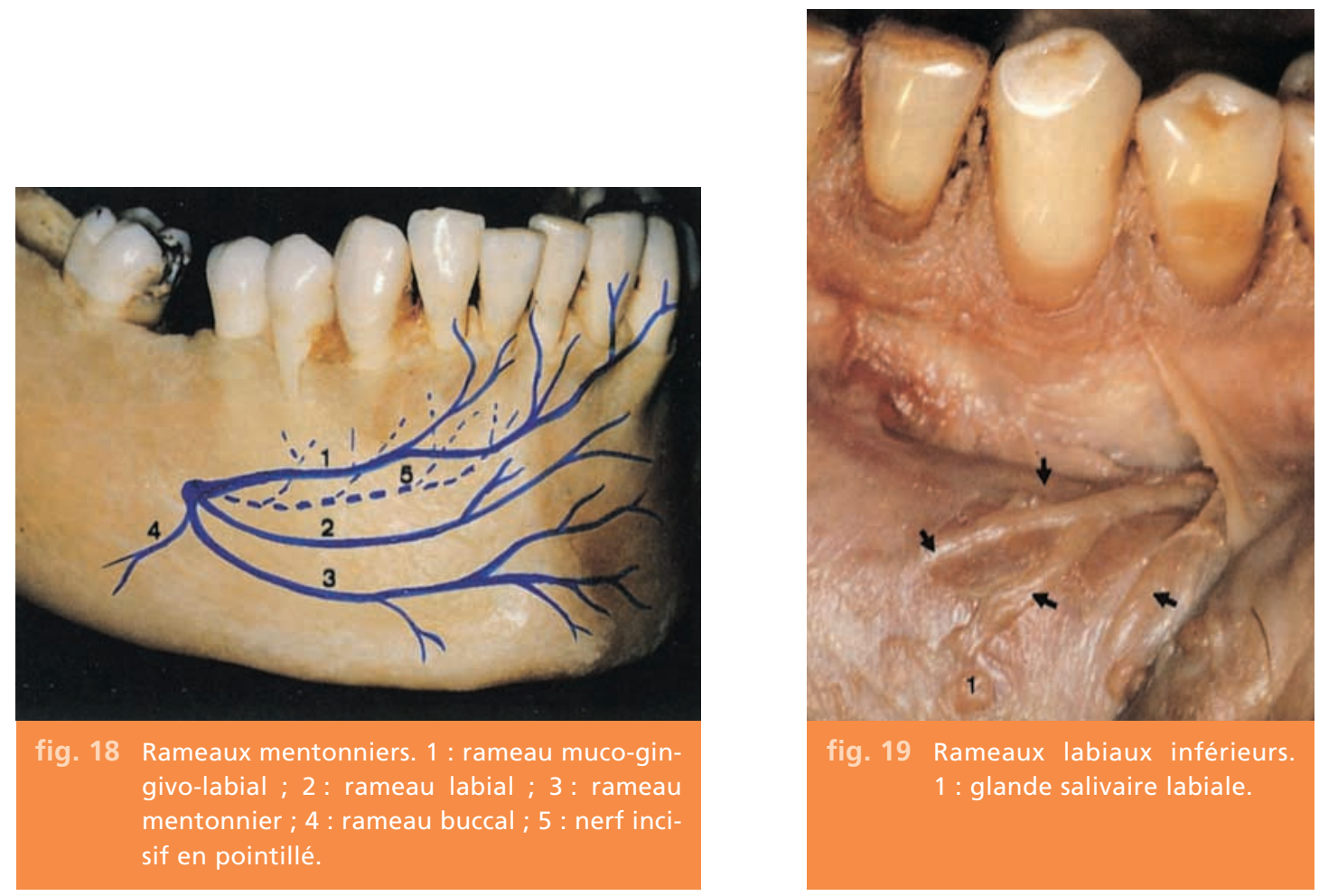

\section{Le nerf lingual}

Dans la région infratemporale, le nerf lingual reçoit une branche collatérale du nerf facial : la corde du tympan (fig. 14). C'est par cette voie que transitent :

- les afférences gustatives en provenance de la muqueuse linguale située en avant du V ;

- les efférences viscérales générales parasympathiques issues du noyau salivaire supérieur destinées aux glandes submandibulaires (sousmaxillaires) et sublinguales.

Le nerf lingual sort de la région infratemporale entre le tendon profond du temporal et le ptérygoïdien médial puis contourne par l'intérieur et par le bas les insertions du ligament ptérygomandibulaire (ptérygo-maxillaire) et du faisceau mylo-hyoïdien du constricteur supérieur du pharynx sur la mandibule. Il est alors recouvert exclusivement par la muqueuse du récessus sublingual latéral et détache un rameau pour la glande submandibulaire avant de s'engager dans le défilé hyoglosse mylo-hyoïdien en compagnie du prolongement antérieur de la glande et du conduit submandibulaire (canal de Wharton), tous deux situés en dedans de lui. Dans la région sublinguale, il chemine entre la paroi latérale de la langue et le complexe salivaire sublingual (glandes sublinguales majeures et mineures), et sous-croise de dehors en dedans le conduit submandibulaire avant de se diviser en plusieurs branches destinées au complexe salivaire et à la muqueuse linguale dans laquelle il se termine (fig. 20).

Le territoire muqueux contrôlé par le nerf lingual comporte la langue en avant du V, la région sublinguale et la paroi alvéolaire médiale du corps mandibulaire.

Le praticien est souvent plus préoccupé par le risque chirurgical que représente le nerf lin- 


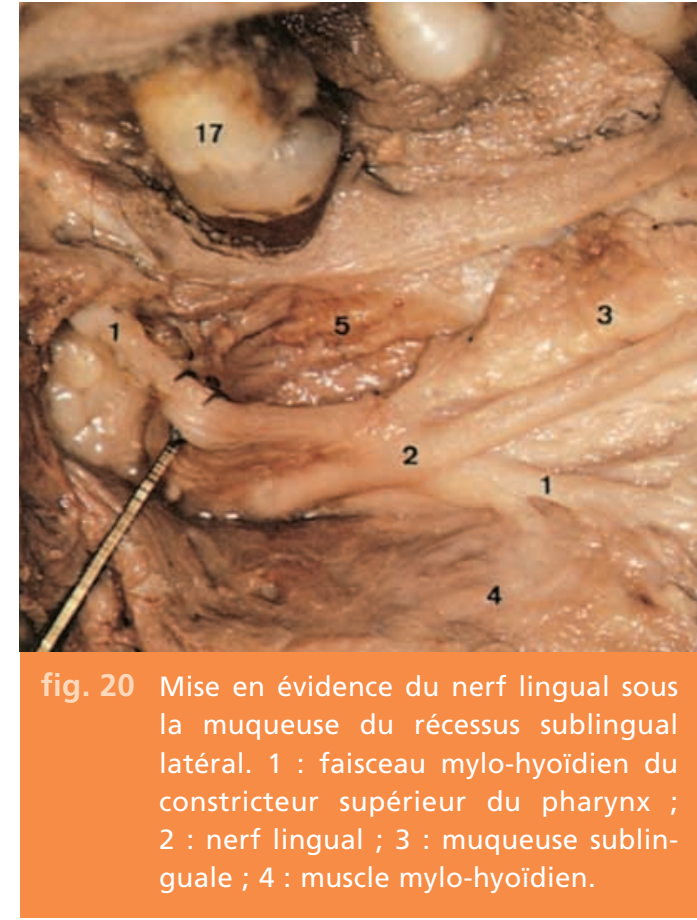

gual dans le secteur de la dent de sagesse que par l'anesthésie de ce tronc nerveux. Celle-ci est obtenue, même si elle n'est pas souhaitée, lors d'une anesthésie à l'épine de Spix, à cause de la proximité des troncs nerveux. Un blocage plus sélectif du nerf lingual peut être envisagé, soit en diminuant d'environ $1 \mathrm{~cm}$ la profondeur de la pénétration de l'aiguille dans la région infratemporale, soit en infiltrant le récessus sublingual latéral (sillon alvéolo-lingual) en regard de la dernière molaire (fig. 21). Remarquons que cette dernière injection peut anesthésier le nerf du mylo-hyoïdien ainsi que le nerf alvéolaire inférieur compte tenu de la relative proximité du canal mandibulaire dans ce secteur et qu'il est de ce fait difficile d'interpréter l'efficacité d'un complément anesthésique à ce niveau en évoquant les suppléances nerveuses (fig. 22).

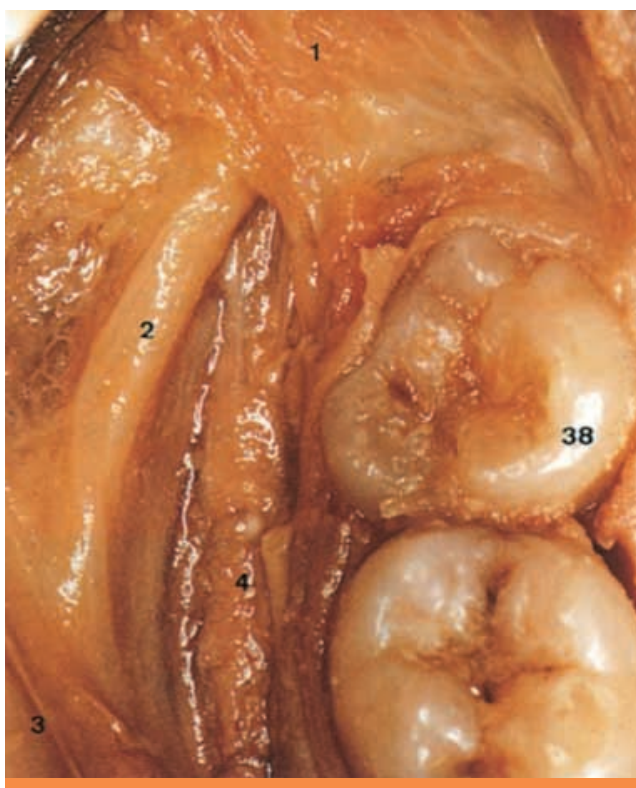

fig. 21 Rapports du nerf lingual dans la région sublinguale. $1:$ nerf lingual ; 2 : canal submandibulaire ; 3 : paroi latérale de la langue ; 4 : glande sublinguale principale.

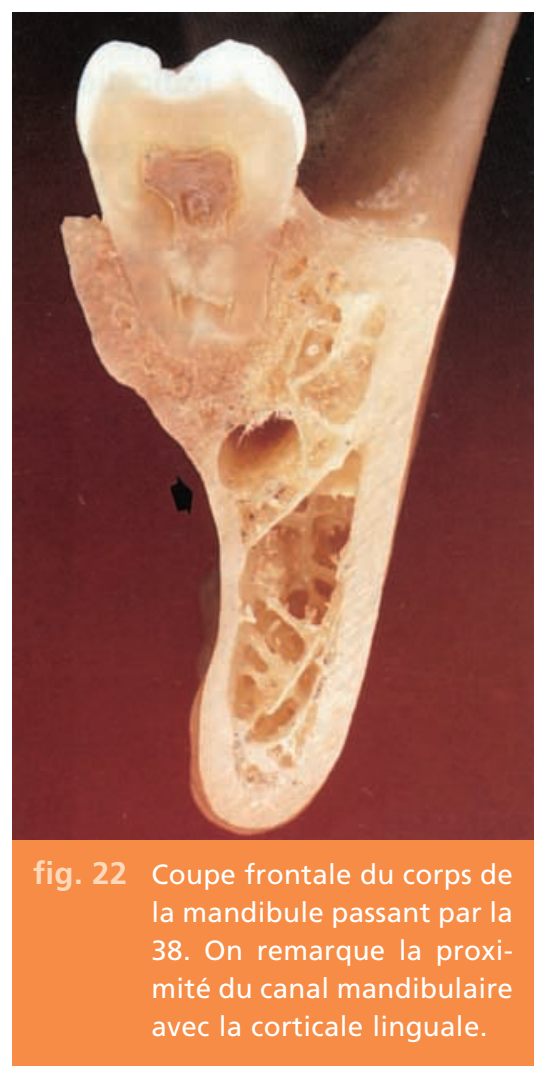




\section{Le nerf auriculo-temporal}

Le nerf auriculo-temporal peut intéresser le praticien concerné par les dysfonctionnements de l'articulation temporo-mandibulaire, puisqu'il véhicule les quatre cinquièmes des afférences en provenance de l'articulation. Son territoire sensitif comprend également le cuir chevelu de la région temporale et une partie de l'auricule (pavillon) : le tragus et la racine de I'hélix. II conduit à la glande parotide les fibres post-ganglionnaires parasympathiques qu'il récupère au contact du ganglion otique où font synapses les fibres issues du noyau salivaire inférieur, associées à leur origine au nerf glosso-pharyngien.

Le nerf auriculo-temporal gagne la parotide en empruntant le défilé ménagé par le col du condyle et le ligament sphéno-mandibulaire (fig. 23). II est alors en rapport avec les vaisseaux maxillaires, puis se dirige vers la région temporale en compagnie des vaisseaux temporaux superficiels.
L'anesthésie du tronc nerveux se pratique en insérant l'aiguille en avant de l'incisure intertragique et en progressant vers le col du condyle qui doit être repéré, puis dépassé en avant et en dedans de $1 \mathrm{~cm}$. L'aspiration, avant $\mathrm{d}^{\prime}$ injecter $1,5 \mathrm{ml}$ de solution anesthésique, est impérative en raison de l'environnement vasculaire. Il faut par contre éviter de tracer pour ne pas anesthésier les rameaux du facial. L'arthro-tomographie, le diagnostic différentiel des algies pré-auriculaires, la réduction d'une luxation discale voire disco-condylienne, constituent les principales indications de cette technique.

- Si la connaissance des voies sensitives trigéminales est essentielle à la pratique de l'anesthésie locale, elle ne constitue pas pour autant une base anatomique suffisante pour maîtriser les techniques. Le praticien doit en effet posséder complètement l'anatomie de la sphère orofaciale à seule fin de reconnaître les structures osseuses et musculaires qu'il est amené à

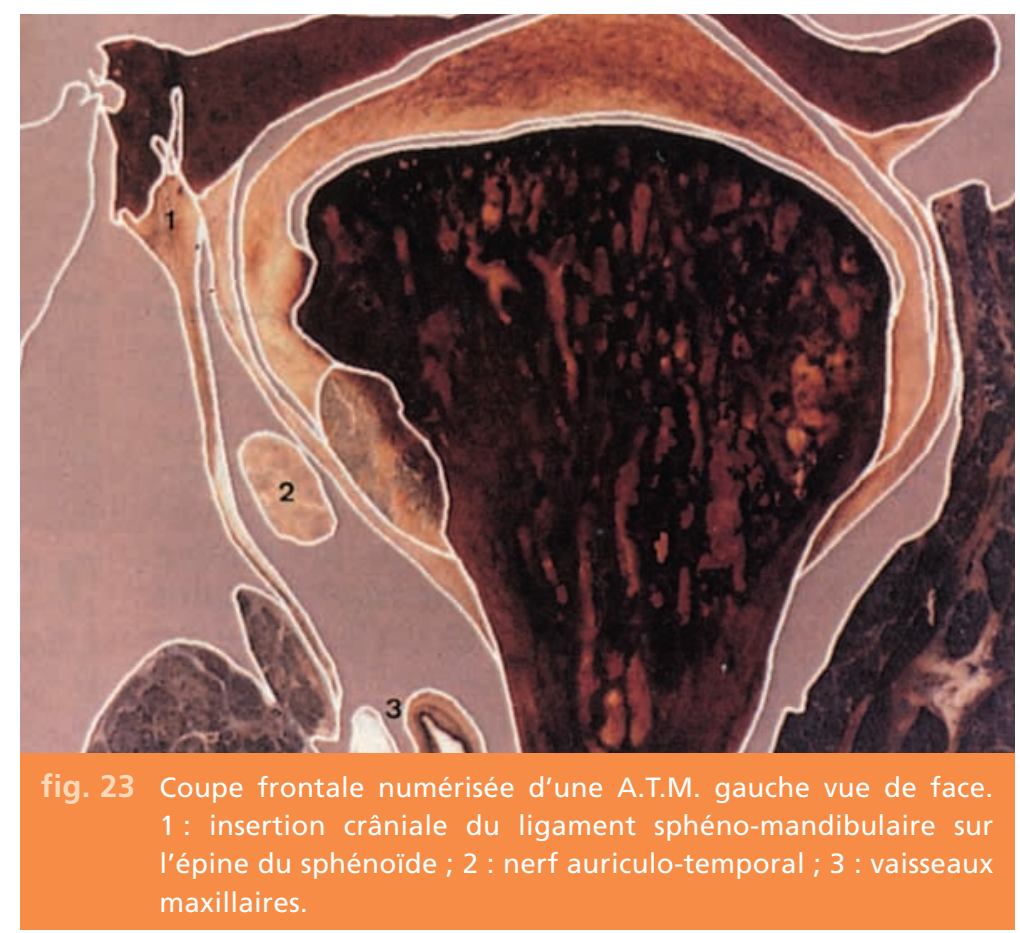


palper, et les éléments vasculaires qu'il risque de pénétrer avec l'aiguille. Rappelons qu'une injection intravasculaire ou intramusculaire constitue un échec anesthésique qui s'accompagne d'effets systémiques immédiats dans le premier cas, et de myalgies dans le second.

Cet article ne prétend pas constituer une nouvelle référence sur l'innervation trigéminale. II est volontairement incomplet puisque limité aux seuls éléments nécessaires au clinicien pour comprendre et pratiquer l'anesthésie locale. II illustre le concept d'anatomie clinique et essaie d'injecter "sans douleur» la Nouvelle Terminologie Anatomique.

Les dissections ont été réalisées par le Professeur Cabrol, à l'École de Chirurgie, 17, rue du Fer-à-Moulin, 75005 Paris.

\section{Bibliographie}

1. Alantar $\mathrm{SA}$, Legens $\mathrm{M}$, Roche $Y$, Carpentier P. L'anesthésie tubérositaire. Actualités Odonto-Stomat 1989;166:335-347.

2. Alantar $S A$, Legens $M$, Roche $Y$, Carpentier $P$. Contribution à la systématisation du nerf mentonnier : incidences chirurgicales. Actualités Odonto-Stomat 1991;176:611-619.

3. Carpentier $P$. L'anesthésie du nerf dentaire inférieur : quelle technique choisir? Acta Stomat Int 1985:2:91-105.

4. Carpentier P. Quoi de neuf sur les techniques anesthésiques ? Rev Odonto-Stomat 1986;6:453-458.

5. Carter RB, Keen EN. The intramandibular cours

of the inferior alveolar nerve. J Anat 1971;108:433-440.
6. Frommer J, Mele FA, Moneroe CW. The possible role of the mylohyoid nerve in mandibular posterior tooth sensation.

J Amer Dent Ass 1972;85:113-117.

7. Gauthier-Lafaye P. Précis d'anesthésie loco-régionale. Paris, Masson, édit 1985

8. Kamina P. Dictionnaire Atlas d'Anatomie. Paris, Maloine, édit 1983.

9. Leblanc A. Imagerie anatomique des nerfs crâniens. Paris, Springer-Verlag, édit 1989.

10. Madeira MC, Percinoto $C$, Silvam L. Clinical significance of supplementary innervation of the lower incisor teeth; a dissection study of the mylohyoid nerve. 1978;46(5):608-613.

11. Ouhayoun JP, Carpentier $P$ Sandot T, Machtou P.
L'anesthésie loco-régionale en odontologie. 1 re partie.

Rev Odonto-Stomat 1981;6:401-414.

12. Ouhayoun JP, Carpentier $P$, Sandot T, Machtou P.

L'anesthésie

loco-régionale

en odontologie.

$2^{\mathrm{e}}$ partie.

Rev Odonto-Stomat 1982;1:9-26.

13. Paturet $G$. Traité

d'anatomie humaine.

Tome 1.

1 re édition. Paris, Masson, édit. 1951.

14. Reynes $P$, Duran $D$, Guiraud P, Guichard M.

Les échecs

de l'anesthésie

des incisives centrales et des molaires mandibulaires. Le problème des suppléances nerveuses. Rev Odonto-Stomat 1983:12:55-61.

15. Rood JP. The analgesia 
and innervation of mandibular teeth. Brit Dent J 1976;140:237-238.

16. Rouvière $\mathrm{H}$ Anatomie humaine descriptive et topographique.

Tome 1.

10 édition

Paris, Masson, édit 1967.
17. Sicher $\mathrm{H}$, Dubrul EL. Oral anatomy.

$7^{\text {th }}$ edition

Saint-Louis, C.V.

Mosby Company, édit 1980 .

18. Sobotta J.

Atlas

d'anatomie humaine.

1 er volume.

$2 \mathrm{e}$ édition.

Paris,
Édition Médicales Internationales,

édit 1985.

19. Sutton RN

The practical significance

of mandibular accessory

foramina.

Aust Dent J

1974;19:167-173.

\section{SUMMARY}

\section{The sensory pathways of the trigeminal nerve: a guide for local anesthesia}

Pierre CARPENTIER

Keywords

- clinical anatomy

- local anesthesia

- trigeminal nerve

- new anatomical

terminology
Knowledge of the nervous pathways is a determining factor for achieving success in the administration of local anesthesia in the oral cavity. This paper presents the basic known data about trigeminal innervation routes using new international anatomic terminology. The data is shown clearly by means of photographs of dry bone segments through which the primary pathways pass. Dissection pictures and anatomic sections are used to complete the schemas. This article illustrates the concept of clinical anatomy, because it is limited to a presentation of only those factors that a clinician needs to know in order to understand and utilize local anesthesia properly. 